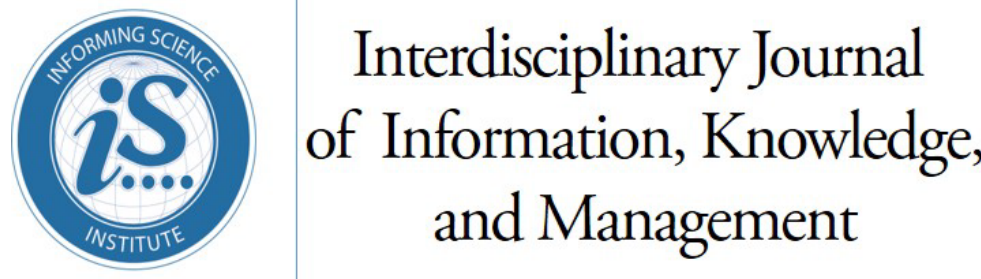

An Official Publication

of the Informing Science Institute

InformingScience.org

IJIKM.org

\title{
Volume 17, 2022 \\ IMPACT OF TEXT DIVERSITY ON REVIEW HELPFULNESS: A TOPIC MODELING APPROACH
}

\begin{tabular}{|c|c|c|}
\hline Lusi Li* & $\begin{array}{l}\text { California State University Los Angeles, } \\
\text { Los Angeles, CA, USA }\end{array}$ & $\underline{\text { lli57@,calstatela.edu }}$ \\
\hline Liuliu Fu & $\begin{array}{l}\text { California State University Los Angeles, } \\
\text { Los Angeles, CA, USA }\end{array}$ & 1fu8@calstatela.edu \\
\hline Wenlu Zhang & $\begin{array}{l}\text { California State University Long Beach, } \\
\text { Long Beach, CA, USA }\end{array}$ & wenlu.zhang@csulb.edu \\
\hline
\end{tabular}

ABSTRACT

Aim/Purpose

In this study, we aim to investigate the impact of an important characteristic of textual reviews - the diversity of the review content on review helpfulness.

Background

Consumer-generated reviews are an essential format of online Word-of-Month that help customers reduce uncertainty and information asymmetry. However, not all reviews are equally helpful as reflected by the varying number of helpfulness votes received by reviews. From consumers' perspective, what kind of content is more effective and useful for making purchase decisions is unclear.

Methodology We use a data set consisting of consumer reviews for laptop products on Amazon from 2014 to 2018. A topic modeling technique is implemented to unveil the hidden topics embedded in the reviews. Based on the extracted topics, we compute the text diversity score of each review. The diversity score measures how diverse the content in a review is compared to other reviews.

Contribution

In the literature, studies have examined various factors that can influence review helpfulness. However, studies that emphasized the information value of textual reviews are limited. Our study contributes to the extant literature of online word-of-mouth by establishing the connection between the diversity of the review content and consumer perceived helpfulness.

Findings

Empirical results show that text diversity plays an important role in consumers' evaluation of whether the review is helpful. Reviews that contain more diverse content tend to be more helpful to consumers. Moreover, we find a negative interaction effect between text diversity and the text depth. This result suggests

Accepting Editor Dimitar Grozdanov Christozov | Received: November 4, 2021 | Revised: February 7 , February 14, 2022 | Accepted: February 17, 2022.

Cite as: Li, L., Fu, L., \& Zhang, W. (2022). Impact of text diversity on review helpfulness: A topic modeling approach. Interdisciplinary Journal of Information, Knowledge, and Management, 17, 87-100. https://doi.org/10.28945/4922

(CC BY-NC 4.0) This article is licensed to you under a Creative Commons Attribution-NonCommercial 4.0 International License. When you copy and redistribute this paper in full or in part, you need to provide proper attribution to it to ensure that others can later locate this work (and to ensure that others do not accuse you of plagiarism). You may (and we encourage you to) adapt, remix, transform, and build upon the material for any non-commercial purposes. This license does not permit you to use this material for commercial purposes. 
Impact of Text Diversity on Review Helpfulness

that text depth and text diversity have a substitution effect. When a review contains more in-depth content, the impact of text diversity is weakened.

Recommendations For consumers to quickly find the informative reviews, platforms should incorfor Practitioners porate measures such as text diversity in the ranking algorithms to rank consumer reviews.

Future Research Future study can extend the current research by examine the impact of text diversity for experienced goods and compare the results with search goods.

Keywords review helpfulness, text diversity, latent dirichlet allocation, online consumer reviews

\section{INTRODUCTION}

Online shopping and e-commerce have been growing rapidly in the past few decades. The total sales of online shopping surpassed $\$ 4.2$ trillion dollars in 2020 (S. Chevalier, 2022). In an online shopping environment, consumers heavily rely on user-generated product reviews to evaluate the quality of the products and mitigate information asymmetry issues. These user-generated online reviews also present a unique opportunity for manufacturers and sellers to receive feedback from consumers in a shorter period of time and with a greater volume than using the traditional survey to collect user feedback. Manufacturers or sellers can extract useful information from these reviews to improve their products or services. However, not all reviews are equally useful and helpful as reflected by the varying number of helpfulness votes received by reviews. As illustrated in Figure 1, consumers can vote for other consumers' reviews if they deem the reviews contain useful information. However, because the platform such as Amazon typically lists the reviews with high votes at the top, some potentially "good" and "useful" reviews that have not received enough votes may be buried under the large volume of reviews. Therefore, it is important to identify potentially helpful reviews that have a great influence on consumers' choice. In the literature, studies have examined various factors that can influence online review valence (i.e., the average review rating) and helpfulness (Ghose \& Ipeirotis, 2010; Liu et al., 2008; Pan \& Zhang, 2011; J. P. Singh et al., 2017). However, studies that emphasized the information value of textual reviews are limited. In this study, we aim to investigate an important characteristic of textual reviews - the diversity of the review content. We consider a review has high text diversity when the review discussed several aspects of the products, or discussed rare aspects of the products (i.e., aspects that are not discussed often in other reviews). In Table 1, the left review discussed several aspects of the product including battery life, screen quality, and keyboard, while the right review mostly discussed the support of the product. Because longer reviews tend to discuss more aspects of the products, we include text depth in our analysis. Text depth measures the length of the review. When two reviews discussed the same aspects of the products, the longer review tends to contain in-depth information about these aspects.

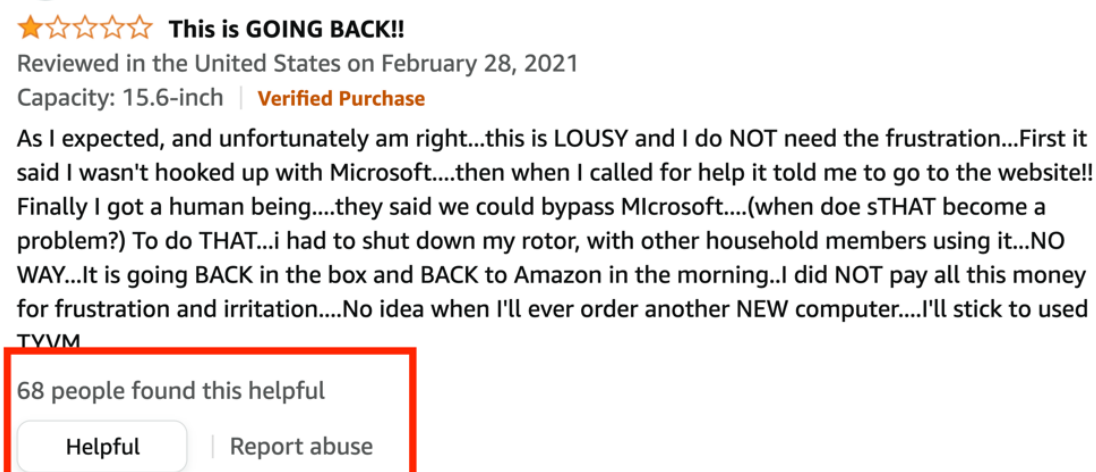

Figure 1: Review Helpfulness 
In our study, topic modeling method is used to extract the hidden aspects (hereafter referred to as topics) embedded in the consumer reviews. Topic modeling is a probabilistic generative model that has been widely explored in text mining. In business research community, more and more studies are employing the topic modeling method to extract useful information from large and unstructured textual data (Bao \& Datta, 2014; Ghose \& Ipeirotis, 2010; Gong et al., 2018; P. V. Singh et al., 2014). In this study, we implement a popular technique in topic modeling - Latent Dirichlet Allocation (LDA) (Blei et al., 2003) to process consumer reviews. We choose LDA because many empirical studies have validated LDA's capability of extracting semantically meaningful topics from textual documents (Boyd-Gradber et al., 2014; Chang et al., 2009; Mimno et al., 2011). LDA is an unsupervised topic model that identifies "abstract topics in a collection of documents" (Aaron et al., 2020), which does not require prior knowledge of the topics in the documents. LDA assumes that each document consists of a set of latent topics, and each topic consists of a set of words. By examining the co-occurrence of words at the review level, the methods can summarize the common topics in the reviews. After extracting the hidden topics, we then use Tao's coefficient measure (Rao, 1982) to calculate the text diversity of the topics. Empirical results show that an increase in text diversity significantly increases the helpfulness of the review. Moreover, we find a negative interaction effect between text diversity and text depth. This result suggests that text depth and text diversity have a substitution effect. When a review contains more in-depth contents, the impact of text diversity is weakened.

Table 1: High Text Diversity versus Low Text Diversity

\begin{tabular}{|c|c|}
\hline $\begin{array}{l}\text { Only had this computer for a few days so perhaps it's } \\
\text { too early to rate but so far I'm very pleased. It's very } \\
\text { slim and lightweight. The set up was super easy. It } \\
\text { boots up almost instantly. The key board is easy to ma- } \\
\text { neuver. The built in mouse pad works extremely well. } \\
\text { The screen quality is very good \& the monitor is } \\
\text { bright. I only use it for basic things like email, social } \\
\text { media, research, on line shopping, etc so it fits my } \\
\text { needs perfectly. The battery life is decent. It came } \\
\text { loaded with Windows } 11 \text { which is great but unfortu- } \\
\text { nately Windows } 11 \text { is not compatible with my printer. } \\
\text { The tradeoff is that Windows } 11 \text { is far superior to Win- } \\
\text { dows } 10 \text { in all other respects. The test will be to see } \\
\text { how long it all holds up. I'm optimistic though! }\end{array}$ & $\begin{array}{l}\text { When I turned it on and signed in I could not make } \\
\text { a desktop icon. Not a big deal, but there was no in- } \\
\text { formation on the internet on how to fix it besides } \\
\text { making a custom shortcut. But in doing so you don't } \\
\text { get the icon. so instead of making a custom icon for } \\
\text { something i bought brand new I contacted support. } \\
\text { it took me almost an hour to actually talk to a person, } \\
\text { it took the person } 12 \text { minutes to before they actually } \\
\text { gave me half a response related to my issue. They } \\
\text { stated that it was a software issue and I needed to } \\
\text { contact their Smart Friend support. You need to pay } \\
\text { for that service. So the brand new laptop i had just } \\
\text { opened without installing anything on it had a soft- } \\
\text { ware problem they wouldn't try and resolve without } \\
\text { me paying for help. }\end{array}$ \\
\hline & with low text diversity \\
\hline
\end{tabular}

\section{LITERATURE REVIEW}

Our paper is closely related to the stream of studies about Online Word-of-Mouth. Since we use text mining techniques to process consumer reviews, we also review the studies in information systems that utilized text mining methods. Lastly, we aim to investigate the information value of consumers reviews by measuring the diversity of review content, thus we review the methods related to text diversity proposed in the previous studies.

\section{ONLINE WORD-OF-MOUTH}

With the popularity of social networks and information sharing, consumers have greater incentives to share their opinions and evaluation about products and services online. Online Word-of-Mouth information has a significant impact on customers' purchase decisions (Chen et al., 2015). Consumergenerated reviews are a popular format of Online Word-of-Mouth. There is a rich body of literature 
Impact of Text Diversity on Review Helpfulness

that examines the impact of consumer reviews on product sales and product rating in different settings (J. A. Chevalier \& Mayzlin, 2006; Chintagunta et al., 2010; Elwalda et al., 2016; Floyd et al., 2014; Zhu \& Zhang, 2010).

Besides sales and rating, another important aspect is perceived helpfulness of a review, which is an indicator of the usefulness and effectiveness of the review. Previous studies have examined various factors that affect the perceived helpfulness. From the reviewer aspect, different types of peripheral cues for reviewer's credibility were reported to affect the consumers' evaluation of review helpfulness (Baek \& Choi, 2012). Forman et al. (2008) found that when the reviewer disclosed their identity information, the review would get more helpful votes than those without reviewer's identity disclosure. Ghose and Ipeirotis (2010) reported a positive relationship between review helpfulness and the total number of past reviews posted by the reviewer. However, Huang et al. (2015) argued that reviewer experience was not a significant predictor of helpfulness. Karimi and Wang (2017) examined reviewer's profile image and found a positive relationship with review helpfulness. From the review aspect, previous studies examined sentiment, stylistic and semantic characteristics of review content. Mudambi and Schuff (2010) reported that the review rating extremity significantly affected the perceived helpfulness of the review, and the magnitude of the effect varied between experience products and search products. C. Wu et al. (2015) observed that reviews with positive sentiment had higher helpful votes than reviews with negative sentiment. Yin et al. (2014) suggested that anxiousness emotion conveyed by the review content leaded to higher perceived helpfulness than anger emotion. Schindler and Bickart (2012) found that stylistic characteristics of review content such as spelling, grammatical errors, expressive slang and humor significantly affected review helpfulness. In addition, review length and readability were reported to affect review helpfulness (Korfiatis et al., 2012; Mudambi \& Schuff, 2010). Hong et al. (2017) provided a comprehensive review of the literature regarding the determinants of review helpfulness from both the reviewer and review perspectives. In this study, we focus on the information value of review content. We measure the diversity of the topics discussed in the reviews. To the best of our knowledge, no previous study has investigated the impact of text diversity on review helpfulness. Empirical results in our study show that text diversity is an indicator of the information value embedded in the reviews and positively associated with review helpfulness.

\section{TEXT MINING}

More and more studies in business research community have recently begun to apply text mining methods to analyze textual data. Gu et al. (2007) used text mining techniques to measure the quality of users' postings in online virtual communities. Ghose and Ipeirotis (2010) extracted text-based features from online reviews by applying text mining techniques. Ghose et al. (2012) used text mining and image classification techniques to recommend products that provide the best value. Moreno and Terwiesch (2014) used text mining techniques to encode the verbal praise left by previous buyers in an online software development community to study the effect of reputation systems on market outcome. In this study, we use a popular technique - Latent Dirichlet Allocation (LDA) topic modeling (Blei et al., 2003) from text mining to process consumer reviews. LDA has been used to automatically extract and summarize information from various types of textual data. P. V. Singh et al. (2014) extracted the textual characteristics related to the quality and the sentiment from blog postings in an enterprise blogosphere. Gong et al. (2018) used LDA to calculate the ambiguity of the search advertising results related to keywords. Bao and Datta (2014) applied LDA on firms' financial reports to simultaneously discover and quantify risk types. LDA is an unsupervised generative probabilistic model which assumes that each consumer review consists of a set of latent topics and each topic consists of a set of words. The process of LDA to generate a consumer review is illustrated as follows. For each review document, a Dirichlet distribution with parameter $\alpha$ is used to generate a T-vector $\theta_{d}$ that represents the review's mixture distribution of topics. Each component of $\theta_{d}$ represents the contribution of a topic $i$ to the review. T represents the total number of topics in the corpus, which is predefined by the user. For each word position in the review, a specific topic $j$ is sampled from $\theta_{d}$ 
as a categorical distribution. For the selected topic $j$, a Dirichlet distribution with parameter $\beta$ is used to generate a V-vector $\theta_{y}$ that represents the document's mixture distribution of topics. V represents the total number of vocabularies in the corpus. Each component of $\theta_{y}$ represents the probability that a topic contains a certain word. A word is sampled according to $\theta_{y}$, and this process continues until all the words in the document are generated. By applying LDA technique, we can get a distribution of topics embedded in each review.

\section{MEASURING TEXT DIVERSITY}

Diversity is an important instrument that has been widely used in sociology and linguistics (Gibbs \& Martin, 1962; Lieberson, 1969). In recommender systems and search engine retrieval, diversified results are considered more informative and useful to help consumer mitigate information overload than highly homogeneous results (Boim et al., 2011; Drosou \& Pitoura, 2012; Hurley \& Zhang, 2011). In the text mining area, several studies have proposed approaches to measure topical diversity of documents, which indicates how diverse a text document is relative to other documents in a corpus (Azarbonyad et al., 2017; Bache et al., 2013; Derzinski \& Rohanimanesh, 2014; Liang et al., 2014). Most of these approaches first extract topics from documents using topic modeling techniques and then estimate the diversity of documents using the extracted topics. Diversity is an important indicator of the information value of a document. In this study, we follow the approach in Bache et al. (2013) to measure the diversity of a review, which used Rao's coefficient (Rao, 1982) to calculate the text diversity after topics are extracted. In particular, given the topic distribution of a review $d$, we define the text diversity as:

$$
\operatorname{Diversity}(d)=\sum_{i=1}^{T} \sum_{j=1}^{T} p_{i d} p_{j d} \delta(i, j)
$$

where T is the number of topics; $p_{i d}$ and $p_{j d}$ are the contribution of topics $i$ and $j$ to review $d$ respectively, and $\delta(i, j)$ is the dissimilarity of topics $i$ and $j$, which is computed as follows:

$$
\delta(i, j)=1-\operatorname{CosineSim}\left(P_{i}, P_{j}\right)
$$

where $P_{i}=\left[p_{i 1}, p_{i 2}, \ldots, p_{i d}\right]$ and $P_{j}=\left[p_{j 1}, p_{j 2}, \ldots, p_{j d}\right] . P_{i}$ and $P_{j}$ are two vectors consisting of the contribution of topic $i$ and topic $j$ in all reviews. $\operatorname{CosineSim}\left(P_{i}, P_{j}\right)$ is the cosine similarity of the two vectors. Cosine similarity is a common measure for similarity between two non-zero vectors. The dissimilarity of the two topics is then the cosine distance of their vectors. Intuitively, text diversity score indicates how dissimilar the topics covered in the review are based on the words assigned to them. If a review contains two topics far away from one another, the review gets a high diversity score. If a review contains relatively close topics, or a large proportion of words is assigned to a single topic, the review gets a lower diversity score.

\section{HYPOTHESIS DEVELOPMENT}

Consumers suffer from heavy information overload and information asymmetry issues in an online environment (Houser \& Wooders, 2006). Effective information can help consumers reduce uncertainty and thus relieve information asymmetry. Consumer-generated reviews are an essential source of information that consumers can easily and readily access. What kind of information is more valuable to consumers to reduce product uncertainty? Ross (2016) defines information as "knowledge, after which one receives and processes, that changes, in an uncertainty changing way, their ex-ante probability distribution regarding a set of propositions or states". In this regard, information is more valuable when the content is less predictable. For instance, reviews that contain different topics from other reviews may change consumers' perception about the products. Fresneda and Gefen (2019) reported that when a review contains different information from the seller's description, the review tends to be more helpful. In addition, several studies reported that the informativeness of reviews are associated with the number of attributes about products discussed in the reviews (Sun et al., 
2019; Srivastava \& Kalro, 2019). In this study, we investigate an important characteristic of consumer reviews - the text diversity of the reviews. The text diversity takes into account both the number of topics and the dissimilarity among topics. Our first hypothesis is:

Hypothesis 1 (H1): Text diversity has a positive relationship with review helpfulness.

The depth of the review content (as measured by the number of words or characters included in a review) also affects the informativeness of the review. When two reviews discussed same aspects of the products, the longer review tends to contain more detailed and in-depth information about these aspects, and thus it is considered more informative (Mudambi \& Schuff, 2010). Liang et al. (2019) reported that review depth has a positive relationship with review helpfulness. Both text diversity and depth can alleviate consumers' uncertainty. However, the relationship between these two are still not clear. In this study, we use the number of words to measure the text depth of a review, and we posit that there is an interaction effect between the text depth and text diversity. We propose the following two hypotheses:

Hypothesis 2 (H2): Text depth has a positive relationship with review helpfulness.

Hypothesis 3 (H3): The impact of text diversity on review helpfulness is moderated by text depth.

In addition to text diversity and text depth, we control the following variables in the model: (1) The posting order. To account for the bias of posting order, we calculate the number of days between the first review of the product and the focal review (Num_Days). We take the log of this variable to take into account the diminishing return of the impact of this variable. (2) The total number of reviews of each product (Num_Reviews). This variable accounts for the bias that different products vary in the number of reviews received. we use the log of this variable in the model. (3) The sentiment of the review. We use the star ratings of a product given by the reviewer (Num_Stars) as a cue of the sentiment of the review. The variable is a 5-scale rating, and a review is considered to convey negative information if the rating is close to 1 . With a rating of 5 , a review is considered to convey positive information. (4) Credibility of the reviewer. We use whether the reviewer is a verified buyer (Verified_Buyer) to account for the reviewer's credibility. (5) Whether a review contains photos of the product (Has_photos). A review with product photos is likely to be more informative to the consumers. Hence, we include this control variable in the model.

\section{METHODS}

\section{DATA}

To test our hypotheses, we use a data set consisting of the consumer reviews on Amazon.com for laptop products from 2014 to 2018 . For each review, the data set comprises product-specific information and review-specific information. We implement the following steps to preprocess the data: (1) removing low-performance products, defined as receiving less than five reviews during the fouryear time window; (2) removing products that contain zero helpful reviews, defined as receiving at least one helpful vote during the four-year time window; and finally (3) removing reviews that are too short (less than 10 words). The resultant data include 1,101 unique products and 26,611 review records. On average, each product gets 24.16 consumer reviews and 9.53 helpful reviews (i.e., reviews that received at least one helpful vote from other consumers). At the review level, on average, each review has 152.07 words and 5.32 helpful votes. The key statistics of variables are reported in Table 2. 


\section{LATENT DIRICHLET ALLOCATION MODEL}

Latent Dirichlet Allocation Model (LDA) is applied to extract meaningful topics from the reviews. First, we convert the consumer reviews into a corpus using the following steps:

1. Remove all the punctuation characters and common stop words in English.

2. Change all the review texts to be lower-cased.

3. Transform review texts to bag-of-words (BoW) representations.

4. Detect bigram and trigram in the review texts.

5. Lemmatize the words to keep only nouns, adjectives, verbs, and adverbs.

Table 2: Summary Statistics

\begin{tabular}{|c|c|c|c|c|c|}
\hline Variable & Obs & Mean & SD & Min & Max \\
\hline Product Level & 1,101 & 24.16 & 42.44 & 5 & 513 \\
\hline Num_Reviews & 1,101 & 9.53 & 15.13 & 1 & 172 \\
\hline Num_HelpfulReviews & 26,611 & 0.39 & 0.48 & 0 & 1 \\
\hline Review Level & 26,611 & 0.04 & 0.12 & 0 & 1 \\
\hline Helpful_Dummy & 26,611 & 0.63 & 0.15 & 0.08 & 1 \\
\hline Helpfulvotes_Ratio & 26,611 & 3.89 & 1.39 & 1 & 5 \\
\hline Diversity & 26,611 & 0.02 & 0.14 & 0 & 1 \\
\hline Num_Stars & 26,611 & 0.80 & 0.39 & 0 & 1 \\
\hline Has_Photos & 26,611 & 258.63 & 243.60 & 0 & 1597 \\
\hline Verified_Buyer & 26,611 & 152.07 & 235.74 & 10 & 4608 \\
\hline Num_Days
\end{tabular}

After processing the data, we use Gensim's LDA module to implement the LDA model for our data set. LDA requires that the user specifies the number of topics. Following the literature, we use the coherence score to determine the optimal number of topics for our consumer reviews corpus (Stevens et al., 2012). The higher the coherence score, the better the LDA model results are. For practical purposes, we consider there are maximum 30 topics in the corpus. Then we run 30 LDA models with the number of topics from 1 to 30 . Figure 2 illustrates the relationship between the coherence score and the number of topics of the LDA model. 15 topics yield the best results based on the coherence score. Therefore, we choose the number of topics to be 15 .

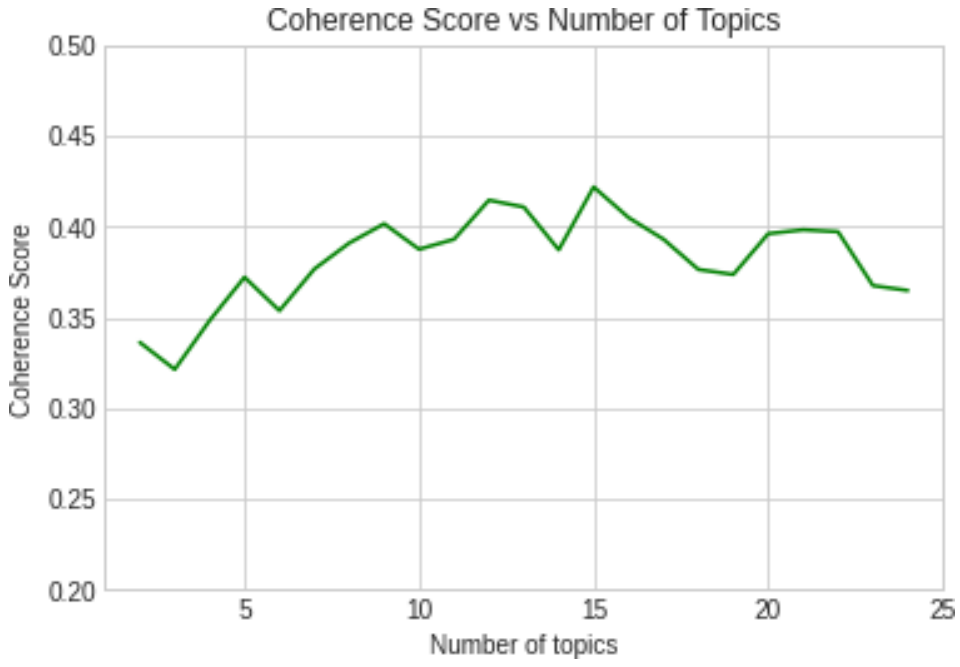

Figure 2: Coherence score and number of Topics 
LDA model generates two outputs: (1) the distribution of topics for each review. (2) the words assigned to each topic. Table 3 lists the 15 topics extracted from the reviews along with the keywords associated with each topic. The topics summarize different aspects of the products that consumers care about. For instance, topic 6 contains keywords such as "Buy, Return, Support", which is related to customer service. Topic 11 contains keywords such as "Battery, Life, Hour", which is related to the quality of the battery. After getting the distribution of topics for each review, we use the formula in Equation (1) to calculate Text Diversity for each review.

Table 3: Topics and Keywords

\begin{tabular}{|l|l|}
\hline Topics & \multicolumn{1}{|c|}{ Keywords } \\
\hline 1 & keyboard, touch, button, key, mouse \\
\hline 2 & Chromebook, chrome, android, extension, device \\
\hline 3 & purchase, good, price, work, product \\
\hline 4 & drive, hard, memory, USB, port \\
\hline 5 & game, play, run, gaming, graphic \\
\hline 6 & buy, return, time, support, work \\
\hline 7 & performance, processor, model, review, much \\
\hline 8 & Screen, good, display, touch, quality \\
\hline 9 & window, update, software, driver, system \\
\hline 10 & ThinkPad, flicker, plate, damage, prompt \\
\hline 11 & battery, life, hour, power, light \\
\hline 12 & video, web, internet, watch, email \\
\hline 13 & cloud, steam, gold, solder, behave \\
\hline 14 & MacBook, apple, pro, air, make \\
\hline 15 & use, buy, love, fast, work \\
\hline
\end{tabular}

\section{RESUlTS OF HYPOTHESIS TESTING}

To examine the impact of text diversity on review helpfulness, we estimated the following empirical model with three rounds of regression analysis.

$$
\begin{aligned}
Y_{i j}=\beta_{0} & +\beta_{1} \text { Diversity }+\beta_{2} \text { Depth }+\beta_{3} \text { Depth } * \text { Diversity } \\
& +\beta_{4} \text { Has_photo }+\beta_{5} \text { Verified_Buyer }+\beta_{6} \text { Num_Stars } \\
& +\beta_{7} \text { log }(\text { Num_Days })+\beta_{8} \text { log }(\text { Num_Reviews })+\varepsilon_{i j}
\end{aligned}
$$

The dependent variable $Y_{i j}$ represents the helpfulness of review $i$ about product $j$. We examined two measures for $Y_{i j}$ : Helpful_Dummy and Helpfulvotes_Ratio. Helpful_Dummy is equal to 1 if a review received at least one helpful vote. This measure can differentiate reviews that never received any votes from reviews that received at least one vote. However, it loses the information about the total number of helpful votes a review received. Therefore, we also used Helpfulvotes_Ratio to measure the review helpfulness. Helpfulvotes_Ratio equals the number of helpful votes received by the review divided by the total number of helpful votes received by the product. To estimate the parameters in Equation (3), we used three regression models. In model (1), we used Helpful_Dummy as the dependent variable. Because Helpful_Dummy is a binary variable, we conducted a logistic regression. In model (2), we used Helpfulvotes_Ratio as the dependent variable and conduct a regular OLS regression. Because the values of Helpfulvotes_Ratio are between 0 and 1, we ran a binomial regression model in model (3). Binomial regression is a common method to model proportion data. The results are summarized in the Table 4. All the analyses are performed in Python 3 using Google Colaboratory.

The results of regression analysis are presented in Table 4. Hypothesis H1 is supported. In particular, text diversity has a significant positive effect on the probability that a review received at least one 
helpful vote (i.e., Helpful_Dummy) and the ratio of the helpful votes among other reviews (i.e., Helpfulvotes_Ratio). This result confirms that consumers care about the diversity of the review content when evaluating the review's usefulness. We also find that the text depth measured by the number of words in a review positively impacts both measures of helpfulness. Therefore, our hypothesis H2 is supported. Moreover, text diversity and text depth have a negative interaction, which supports hypothesis H3. This suggests that consumers care about both the breadth and depth of the review contents. If a review contains in-depth information, consumers are more lenient about the diversity of the contents. Additionally, whether the reviewer is verified (Verified_Buyer) and whether the review contains photos (i.e., Has_Photos) are positively correlated with both measures of review helpfulness. The posting order measured by the number of days since the first review negatively affects both review helpfulness measures. However, we find that rating does not have a significant impact on helpfulness votes ratio but has a negative impact with a low significance level on whether a review receives at least one helpful vote. In previous studies, the effect of ratings on helpfulness was not conclusive (Pan \& Zhang, 2011; Racherla \& Friske, 2012; P. F. Wu, 2013).

Table 4: Results of Regression Analyses

\begin{tabular}{|l|l|l|l|}
\hline & \multicolumn{1}{|c|}{ Model (1) } & \multicolumn{1}{|c|}{ Model (2) } & \multicolumn{1}{c|}{ Model (3) } \\
\hline Dependent Varia- & Helpful_Dummy & Helpfulvotes_Ratio & Helpfulvotes_Ratio \\
ble methods & Logistic Regression & Linear Regression & Binomial Regression \\
\hline Diversity & $2.822^{* * *}$ & $0.021 * * *$ & $2.704^{* * *}$ \\
& $(0.149)$ & $(0.006)$ & $(0.306)$ \\
\hline Depth & $0.019^{* * *}$ & $0.0003 * * *$ & $0.010 * * *$ \\
& $(0.001)$ & $(0.00004)$ & $(0.001)$ \\
\hline Diversity*Depth & $-0.018^{* * *}$ & $-0.0002 * * *$ & $-0.010 * * *$ \\
& $(0.001)$ & $(0.00005)$ & $(0.002)$ \\
\hline Has_Photos & $1.512 * * *$ & $0.049 * * *$ & $0.739 * * *$ \\
& $(0.128)$ & $(0.006)$ & $(0.160)$ \\
\hline Verified_Buyer & $0.097 *$ & $0.013 * * *$ & $0.278^{* * *}$ \\
& $(0.040)$ & $(0.002)$ & $(0.079)$ \\
\hline Num_Stars & $-0.116^{* * *}$ & -0.0003 & 0.008 \\
& $(0.011)$ & $(0.00001)$ & $(0.026)$ \\
\hline log(Num_Reviews $)$ & $0.033^{* *}$ & $-0.022 * * *$ & $-0.906 * * *$ \\
& $(0.012)$ & $(0.001)$ & $(0.038)$ \\
\hline log(Num_Days $)$ & $-0.653 * * *$ & $-0.033 * * *$ & $-0.455^{* * *}$ \\
& $(0.014)$ & $(0.001)$ & $0.017)$ \\
\hline
\end{tabular}

Significance levels: $* * *$ at $0.001, * *$ at $0.01, *$ at 0.05 .

Robust standard errors are reported in parentheses.

\section{DISCUSSION}

The results of our study show that text diversity plays an important role in consumers' evaluation of whether a review is helpful. Reviews that contain more diverse content tend to be more helpful, thus more informative to consumers. The diversity is measured from two aspects: the breadth of the information (i.e., number of topics) and the uniqueness of the information (i.e., dissimilarity among topics). Previous research that studied the relationship between review text and helpfulness primarily focused on the sentiment conveyed by the text or the stylistic characteristics of the text (Mudambi \& Schuff, 2010; Schindler \& Bickart, 2012; C. Wu et al., 2015; Yin et al., 2014). Our findings add to previous research by showing a new antecedent of review helpfulness that deals with the semantic characteristics of reviews - the diversity of the review content compared to other reviews. Consistent with the findings in previous studies, we show that text depth is positively associated with review 
helpfulness (Liang et al., 2019; Mudambi \& Schuff, 2010). However, our analysis reveals that the positive effect of text depth is moderated by text diversity. In particular, we find a negative interaction effect between text diversity and text depth. While both text depth and text diversity affect the informativeness of reviews and help customers reduce the product uncertainty, text depth and text diversity have a substitution effect. When consumers are satisfied with the depth of the information, they are more lenient about the diversity of the information.

\section{CONCLUSION}

Customer reviews carry important signals from consumers to sellers and manufacturers. Proper extraction of information from the reviews helps sellers better understand what consumers care about and further make appropriate decisions in improving products. From consumers' perspective, what kind of information is more effective for them to make purchase decisions is unclear. This study uses the topic modeling technique to extract the hidden topics underlying the last volume of review texts. Based on the extracted topics, we compute the text diversity score of each review. The diversity score measures both the breadth of the information (i.e., number of topics) and the uniqueness of the information (i.e., dissimilarity among topics) in a review. We show that diversity is an important indicator of the informativeness and effectiveness of a review.

This study contributes to the extant literature of online word-of-mouth by establishing the connection between the diversity of the review content and consumer perceived helpfulness. This study also makes several practical contributions. First, manufacturers or sellers can apply topic modeling technique and text diversity measure to obtain the distribution of topics in the consumer reviews and the dissimilarity among topics. In doing so, they can quickly grasp the aspects of the products that consumers care about most. Second, for consumers to quickly find the informative reviews, platforms should incorporate measures such as text diversity in the ranking algorithms to rank consumer reviews.

Like other research studies, this study is subject to some limitations. First, we use the consumer reviews for laptop category in our empirical study. Laptops belong to search goods because it is relatively easy to find product features online. Future study can extend the current research by examine the impact of text diversity for experienced goods and compare the results with search goods. Second, we use LDA topic modeling technique to extract the hidden topics in reviews and the cosine similarity in calculating the diversity. Future study can explore other topic modeling methods and similarity measures.

\section{REFERENCES}

Aaron, J., Benjamin, J., \& Christopher, K. (2020). The unsupervised learning workshop. Packt Publishing.

Azarbonyad, H., Dehghani, M., Kenter, T., Marx, M., Kamps, J., \& de Rijke, M. (2017, April). Hierarchical reestimation of topic models for measuring topical diversity. In J. M. Jose, C. Hauff, I. S. Altıngovde, D. Song, D. Albakour, S. Watt, \& J. Tait (Eds.), Advances in Information Retrieval: Proceedings of the 39 th European Conference on Information Retrieval Research (pp. 68-81). Aberdeen, United Kingdom: Springer, Cham. https://doi.org/10.1007/978-3-319-56608-5 6

Bache, K., Newman, D., \& Smyth, P. (2013, August). Text-based measures of document diversity. In R. Ghani, T. E. Senator, P. Bradley, R. Parekh, J. He (Eds.), Proceedings of the 19th ACM SIGKDD International Conference on Knowledge Discovery and Data Mining (pp. 23-31). Chicago Illinois, USA: ACM. https://doi.org/10.1145/2487575.2487672

Baek, H., Ahn, J., \& Choi, Y. (2012). Helpfulness of online consumer reviews: Readers' objectives and review cues. International Journal of Electronic Commerce, 17(2), 99-126. https://doi.org/10.2753/JEC10864415170204

Bao, Y., \& Datta, A. (2014). Simultaneously discovering and quantifying risk types from textual risk disclosures. Management Science, 60(6), 1371-1391. https://doi.org/10.1287/mnsc.2014.1930 
Blei, D. M., Ng, A. Y., \& Jordan, M. I. (2003). Latent Dirichlet allocation. Journal of Macbine Learning Research, 3(Jan), 993-1022. https://www.jmlr.org/papers/volume3/blei03a/blei03a.pdf

Boim, R., Milo, T., \& Novgorodov, S. (2011, October). Diversification and refinement in collaborative filtering recommender. In B. Berendt, A. de Vries, W. Fan, C. Macdonald, I. Ounis, I. Ruthven (Eds.), Proceedings of the 20th ACM International Conference on Information and Knowledge Management (pp. 739-744). Glasgow Scotland, UK: ACM. https://doi.org/10.1145/2063576.2063684

Boyd-Graber, J., Mimno, D., \& Newman, D. (2014). Care and feeding of topic models: Problems, diagnostics, and improvements. Handbook of mixed membership models and their applications (pp. 225-254). http:// www.people.fas.harvard.edu/ airoldi/pub/books/b02.AiroldiBleiEroshevaFienberg2014HandbookMMM/Ch12 MMM2014.pdf

Chang, J., Boyd-Graber, J. L., Gerrish, S., Wang, C., \& Blei, D. M. (2009, December). Reading tea leaves: How humans interpret topic models. In Y. Bengio, D. Schuurmans, J. D. Lafferty, C. K. I. Williams, A. Culotta (Eds.), Proceedings of the 22nd International Conference on Neural Information Processing Systems (pp. 288-296). Vancouver BC, Canada: ACM. https://dl.acm.org/doi/10.5555/2984093.2984126

Chen, C. H., Nguyen, B., Klaus, P., \& Wu, M. S. (2015). Exploring electronic word-of-mouth (eWOM) in the consumer purchase decision-making process: The case of online holidays - Evidence from United Kingdom (UK) consumers. Journal of Travel \& Tourism Marketing, 32 (8), 953-970. https://doi.org/10.1080/10548408.2014.956165

Chevalier, J. A., \& Mayzlin, D. (2006). The effect of word of mouth on sales: Online book reviews. Journal of Marketing Research, 43(3), 345-354. https://doi.org/10.1509/jmkr.43.3.345

Chevalier, S. (2022, February 04). Retail e-commerce sales worldwide from 2014 to 2025 (in billion US dollars). Statista. https://www.statista.com/statistics/379046/worldwide-retail-e-commerce-sales/

Chintagunta, P. K., Gopinath, S., \& Venkataraman, S. (2010). The effects of online user reviews on movie box office performance: Accounting for sequential rollout and aggregation across local markets. Marketing Science, 29(5), 944-957. https://doi.org/10.1287/mksc.1100.0572

Derezinski, M., \& Rohanimanesh, K. (2014, December). An information theoretic approach to quantifying text interestingness. In NIPS 2014 Workshop on Modern Machine Learning and Natural Language Processing. https://users.soe.ucsc.edu/ mderezin/interestingness.pdf

Drosou, M., \& Pitoura, E. (2012, November). Disc diversity: Result diversification based on dissimilarity and coverage. Proceedings of the VLDB Endowment, 6(1), 13-24. https://doi.org/10.14778/2428536.2428538

Elwalda, A., Lü, K., \& Ali, M. (2016). Perceived derived attributes of online customer reviews. Computers in Human Behavior, 56, 306-319. https://doi.org/10.1016/j.chb.2015.11.051

Floyd, K., Freling, R., Alhoqail, S., Cho, H. Y., \& Freling, T. (2014). How online product reviews affect retail sales: A meta-analysis. Journal of Retailing, 90(2), 217-232. https://doi.org/10.1016/j.jretai.2014.04.004

Forman, C., Ghose, A., \& Wiesenfeld, B. (2008). Examining the relationship between reviews and sales: The role of reviewer identity disclosure in electronic markets. Information Systems Research, 19(3), 291-313. https://doi.org/10.1287/isre.1080.0193

Fresneda, J. E., \& Gefen, D. (2019). A semantic measure of online review helpfulness and the importance of message entropy. Decision Support Systems, 125, 113117. https://doi.org/10.1016/i.dss.2019.113117

Ghose, A., \& Ipeirotis, P. G. (2010). Estimating the helpfulness and economic impact of product reviews: Mining text and reviewer characteristics. IEEE Transactions on Knowledge and Data Engineering, 23(10), 1498-1512. https://doi.org/10.1109/TKDE.2010.188

Ghose, A., Ipeirotis, P. G., \& Li, B. (2012). Designing ranking systems for hotels on travel search engines by mining user-generated and crowdsourced content. Marketing Science, 31(3), 493-520.

https://doi.org/10.1287/mksc.1110.0700

Gibbs, J. P., \& Martin, W. T. (1962). Urbanization, technology, and the division of labor: International patterns. American Sociological Review, 27(5), 667-677. https://doi.org/10.2307/2089624 
Impact of Text Diversity on Review Helpfulness

Gong, J., Abhishek, V., \& Li, B. (2018). Examining the impact of keyword ambiguity on search advertising performance: A topic model approach. MIS Quarterly, 42(3), 805-829. https://doi.org/10.25300/MISQ/2018/14042

Gu, B., Konana, P., Rajagopalan, B., \& Chen, H. W. M. (2007). Competition among virtual communities and user valuation: The case of investing-related communities. Information Systems Research, 18 (1), 68-85. https://doi.org/10.1287/isre.1070.0114

Hong, H., Xu, D., Wang, G. A., \& Fan, W. (2017). Understanding the determinants of online review helpfulness: A meta-analytic investigation. Decision Support Systems, 102, 1-11. https://doi.org/10.1016/j.dss.2017.06.007

Houser, D., \& Wooders, J. (2006). Reputation in auctions: Theory, and evidence from eBay. Journal of Economics \& Management Strategy, 15(2), 353-369. https://doi.org/10.1111/j.1530-9134.2006.00103.x

Huang, A. H., Chen, K., Yen, D. C., \& Tran, T. P. (2015). A study of factors that contribute to online review helpfulness. Computers in Human Behavior, 48, 17-27. https://doi.org/10.1016/j.chb.2015.01.010

Hurley, N., \& Zhang, M. (2011). Novelty and diversity in top-n recommendation - Analysis and evaluation. ACM Transactions on Internet Technology (TOIT), 10(4), Article 14. https://doi.org/10.1145/1944339.1944341

Karimi, S., \& Wang, F. (2017). Online review helpfulness: Impact of reviewer profile image. Decision Support Systems, 96, 39-48. https://doi.org/10.1016/j.dss.2017.02.001

Korfiatis, N., García-Bariocanal, E., \& Sánchez-Alonso, S. (2012). Evaluating content quality and helpfulness of online product reviews: The interplay of review helpfulness vs. review content. Electronic Commerce Research and Applications, 11 (3), 205-217. https://doi.org/10.1016/j.elerap.2011.10.003

Liang, S., Ren, Z., \& De Rijke, M. (2014, August). Personalized search result diversification via structured learning. In Proceedings of the 20th ACM SIGKDD International Conference on Knowledge Discovery and Data Mining (pp. 751-760). New York, USA: ACM. https://doi.org/10.1145/2623330.2623650

Liang, S., Schuckert, M., \& Law, R. (2019). How to improve the stated helpfulness of hotel reviews? A multilevel approach. International Journal of Contemporary Hospitality Management, 31(2), 953-977. https://doi.org/10.1108/IJCHM-02-2018-0134

Lieberson, S. (1969). Measuring population diversity. American Sociological Review, 34(6), 850-862. https://doi.org/10.2307/2095977

Liu, Y., Huang, X., An, A., \& Yu, X. (2008, December). Modeling and predicting the helpfulness of online reviews. In Proceedings of the 2008 Eighth IEEE International Conference on Data Mining (pp. 443-452). Pisa, Italy: IEEE. https://doi.org/10.1109/ICDM.2008.94

Mimno, D., Wallach, H., Talley, E., Leenders, M., \& McCallum, A. (2011, July). Optimizing semantic coherence in topic models. In Proceedings of the 2011 Conference on Empirical Methods in Natural Language Processing (pp. 262-272). Edinburgh, Scotland, UK: Association for Computational Linguistics. https://aclanthology.org/D11-1024.pdf

Moreno, A., \& Terwiesch, C. (2014). Doing business with strangers: Reputation in online service marketplaces. Information Systems Research, 25(4), 865-886. https://doi.org/10.1287/isre.2014.0549

Mudambi, S. M., \& Schuff, D. (2010). Research note: What makes a helpful online review? A study of customer reviews on Amazon.com. MIS Quarterly, 34(1), 185-200. https://doi.org/10.2307/20721420

Pan, Y., \& Zhang, J. Q. (2011). Born unequal: A study of the helpfulness of user-generated product reviews. Journal of Retailing, 87(4), 598-612. https://doi.org/10.1016/j.jretai.2011.05.002

Racherla, P., \& Friske, W. (2012). Perceived usefulness of online consumer reviews: An exploratory investigation across three services categories. Electronic Commerce Research and Applications, 11(6), 548-559. https://doi.org/10.1016/j.elerap.2012.06.003

Rao, C. R. (1982). Diversity and dissimilarity coefficients: A unified approach. Theoretical Population Biology, 21(1), 24-43. https://doi.org/10.1016/0040-5809(82)90004-1 
Ross, J. F. (2016). The information content of accounting reports: An information theory perspective. Information, 7(3), 48. https://doi.org/10.3390/info7030048

Schindler, R. M., \& Bickart, B. (2012). Perceived helpfulness of online consumer reviews: The role of message content and style. Journal of Consumer Behaviour, 11(3), 234-243. https://doi.org/10.1002/cb.1372

Singh, J. P., Irani, S., Rana, N. P., Dwivedi, Y. K., Saumya, S., \& Roy, P. K. (2017). Predicting the helpfulness of online consumer reviews. Journal of Business Research, 70, 346-355.

https://doi.org/10.1016/i.jbusres.2016.08.008

Singh, P. V., Sahoo, N., \& Mukhopadhyay, T. (2014). How to attract and retain readers in enterprise blogging? Information Systems Research, 25(1), 35-52. https://doi.org/10.1287/isre.2013.0509

Srivastava, V., \& Kalro, A. D. (2019). Enhancing the helpfulness of online consumer reviews: The role of latent (content) factors. Journal of Interactive Marketing, 48, 33-50. https://doi.org/10.1016/j.intmar.2018.12.003

Stevens, K., Kegelmeyer, P., Andrzejewski, D., \& Buttler, D. (2012, July). Exploring topic coherence over many models and many topics. In Proceedings of the 2012 Joint Conference on Empirical Methods in Natural Language Processing and Computational Natural Language Learning (pp. 952-961). Jeju Island, Korea: Association for Computational Linguistics. https://aclanthology.org/D12-1087.pdf

Sun, X., Han, M., \& Feng, J. (2019). Helpfulness of online reviews: Examining review informativeness and classification thresholds by search products and experience products. Decision Support Systems, 124, 113099. https://doi.org/10.1016/j.dss.2019.113099

Wu, C., Che, H., Chan, T. Y., \& Lu, X. (2015). The economic value of online reviews. Marketing Science, 34(5), 739-754. https://doi.org/10.1287/mksc.2015.0926

Wu, P. F. (2013). In search of negativity bias: An empirical study of perceived helpfulness of online reviews. Psychology \& Marketing, 30(11), 971-984. https://doi.org/10.1002/mar.20660

Yin, D., Bond, S. D., \& Zhang, H. (2014). Anxious or angry? Effects of discrete emotions on the perceived helpfulness of online reviews. MIS Quarterly, 38(2), 539-560.

https://doi.org/10.25300/MISQ/2014/38.2.10

Zhu, F., \& Zhang, X. (2010). Impact of online consumer reviews on sales: The moderating role of productand consumer characteristics. Journal of Marketing, 74(2), 133-148. https://doi.org/10.1509/jm.74.2.133

\section{AUTHORS}

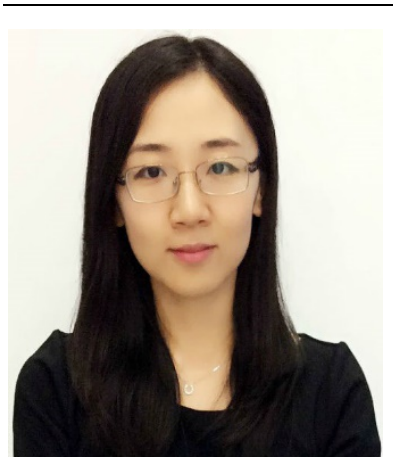

Lusi Li is an Assistant Professor of Information Systems at California State University LA. She received her Ph.D. in Management Science from Naveen Jindal School of Management at the University of Texas at Dallas. Her research interests include economics of information technology, use of recommender systems, online reviews, sharing economy, and healthcare analytics. 


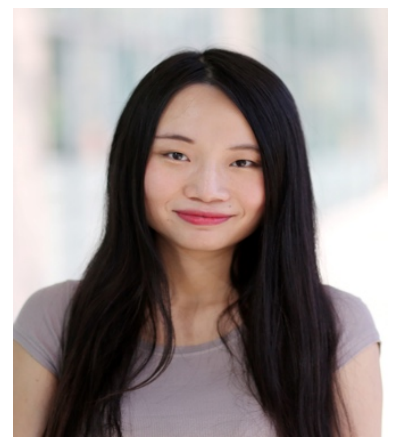

Liuliu Fu is an Assistant Professor of Information Systems at California State University LA. She got my bachelor's degree in Electronic Engineering from Tsinghua University, China, and Ph.D. from Old Dominion University. Her research interests are in data mining, big data, and healthcare system.

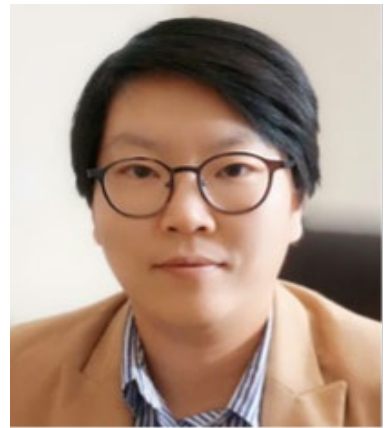

Wenlu Zhang is an Assistant Professor at the Computer Engineering and Computer Science Department at California State University, Long Beach. She received her Ph.D. in Computer Science from Old Dominion University in 2016. Her research interests include machine learning, data mining, computational biology and computational neuroscience. Dr. Zhang received an Outstanding Research Assistant Award in Old Dominion University. 